\title{
Post-extrasystolic potentiation without a compensatory pause in normal and diseased hearts
}

\author{
Paul J P Kuijer, Tjeerd van der Werf, Frits L Meijler
}

\begin{abstract}
Variables derived from left ventricular volume were used to study post-extrasystolic potentiation. Left ventriculograms were obtained from 11 healthy individuals and 49 patients with coronary heart disease (30 with a previous myocardial infarction and 19 without any signs of myocardial damage). Postextrasystolic potentiation was induced by a regularly driven right atrial rhythm that was interrupted by one atrial extrasystole in such a way that the post-extrasystolic $R R$ interval was kept equal to the basic RR interval. The left ventricular end diastolic volumes of the pre-extrasystolic and post-extrasystolic beats were equal. In all groups there was evidence of post-extrasystolic potentiation in one or more of the indices of left ventricular function (ejection fraction, mean normalised systolic ejection rate, end systolic volume, and stroke volume). Potentiation was especially evident in patients with left ventricular damage; this suggests that a compensating mechanism is an intrinsic property of the myocardium.

The Frank-Starling mechanism does not contribute to the increased performance of the post-extrasystolic beat in normal individuals or in patients with coronary artery disease.
\end{abstract}

A century ago Langendorff found that the force of contraction was stronger in the beat that followed an extrasystole. ${ }^{1}$ Hoffman et al called this phenomenon post-extrasystolic potentiation. ${ }^{2}$ Cohn in an editorial cited several investigators ${ }^{3}$ and attributed post-extrasystolic potentiation in vivo to the prolonged pause that follows the extrasystole, which would lead to increased ventricular filling, ${ }^{4-6}$ and reduced aortic impedance (resulting from a decline in aortic pressure during that compensatory pause). ${ }^{7}$ A third mechanism, which was seldom taken into account, is the phenomenon called "rest contraction". 8-10 This phenomenon, which is part of the so-called restitution curve, ${ }^{11}$ is present after a compensatory pause.

Sung et al showed post-extrasystolic potentiation in healthy individuals without an increased end diastolic volume as a contributing factor. ${ }^{12}$ Wisenbaugh et al found evidence of post-extrasystolic potentiation without an increased post-extrasystolic end diastolic volume in patients with volume overload caused by chronic valve regurgitation. ${ }^{13}$ In these studies the consequence of the compensatory pause-that is the effect of the "rest contraction"- - on the size of the post-extrasystolic beat was not taken into account.

Van der Werf et al showed that human postextrasystolic potentiation can be elicited without a compensatory pause. ${ }^{14}$ They provoked controlled post-extrasystolic potentiation by altering the RR interval and by use of pressurederived indices of contractility in normal and diseased hearts.

We used volume derived indices to investigate post-extrasystolic potentiation after a post-extrasystolic $R R$ interval without a compensatory pause in healthy individuals and in patients with coronary heart disease.

\section{Patients and methods}

PATIENTS

We studied 60 patients. Each patient gave informed consent. The patients were divided into three groups: 11 with atypical chest pain, normal coronary arteries, and normal left ventricular pressure and volumes (group 1); 19 patients with typical angina pectoris without evidence of previous myocardial infarction (group 2); and 30 patients with enzymatic and electrocardiographic evidence of an earlier transmural myocardial infarction (group 3). We excluded patients with other forms of cardiac disease and patients who had had cardiac surgery.

\section{STIMULATION PROCEDURE}

We used a computed controlled stimulator to ensure that the duration of the post-extrasystolic RR interval was equal to the basic RR interval. ${ }^{14}$ The stimulation pattern was applied to the right atrium in such a way that variations in atrioventricular nodal delay caused by changes in the extrasystolic PR interval were compensated for. A test series of atrial stimulation pulses was generated and $R R$ intervals were measured on line by computer. PR intervals were measured and atrial stimulus intervals were corrected by iteration so that the length of the post-extrasystolic RR interval was equal (inaccuracy $<1 \%$ ) to that of the preextrasystolic cardiac cycle (figure). A stimulation series consisted of 20 basic intervals interrupted by one extrasystolic interval. The post-extrasystolic beat $(P)$ was compared with the pre-extrasystolic or control beat (C).

QUANTITATIVE LEFT VENTRICULOGRAPHY

Left ventriculography (50 frames) was 


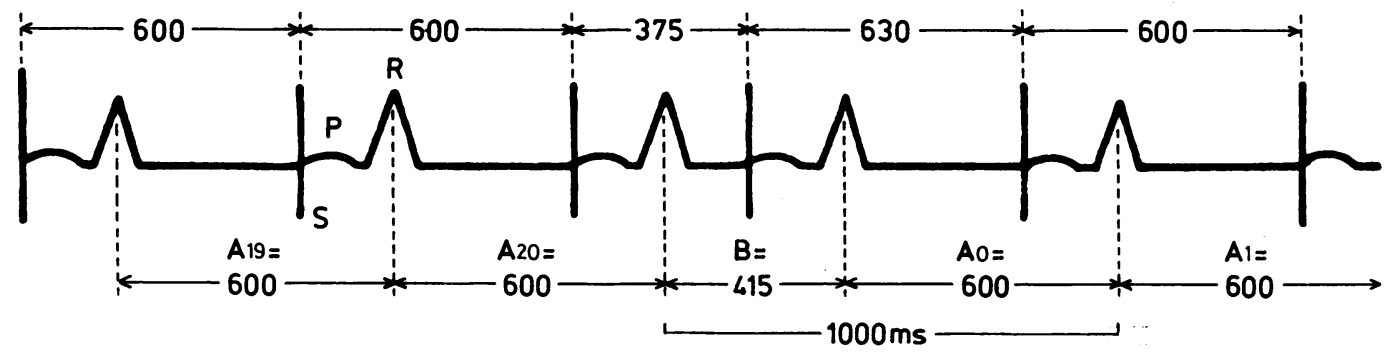

Schematic presentation of the stimulation procedure used. Time intervals between stimulation spikes $(S), P$ waves, and $R$ waves are shown in ms. After 20 basic stimuli a shorter stimulus-stimulus interval ( $375 \mathrm{~ms}$ ) was applied resulting in an $R R$ interval of $415 \mathrm{~ms}(B)$. The difference between the RR interval and the stimulus-stimulus interval is caused by prolongation of the PR interval preceding the extrasystole. To correct for this prolongation, the post-extrasystolic stimulus-stimulus interval was chosen in such a way (630 ms) that it results in a post-extrasystolic RR interval ( $A 0)$ equal to the basic interval ( $A$ 20).

performed in the frontal and left lateral projection after injection of $40-56 \mathrm{ml}$ metrizoate (Isopaque). The pulse for each cineframe was recorded on paper together with the electrocardiogram. We chose the peak of the $R$ wave to mark end diastole. End systole was more difficult to identify, particularly in patients with coronary heart disease. ${ }^{15}$ The frame taken $80 \mathrm{~ms}$ before the opening of the mitral valve was taken as end systole. The end diastolic and end systolic ventricular silhouettes were traced by hand and biplane left ventricular volumes were calculated by Dodge's arealength method. ${ }^{16}$ Stroke volume, ejection fraction, and mean normalised systolic ejection rate were calculated from the volumes. The ejection rate was calculated by dividing the stroke volume by the duration of the ejection period. Because Van der Werf et al found no significant difference in end diastolic aortic pressure between pre-extrasystolic and post-extrasystolic beats, using an identical stimulation protocol, ${ }^{14}$ we did not measure aortic pressures in our study. Data are presented as mean (SD).

We used the Wilcoxon matched pairs signed ranked method for statistical analysis. Differences between groups were regarded as significant when $\mathrm{p}$ was $<0.05$.

\section{INTRA-OBSERVER VARIABILITY}

End diastolic and end systolic volumes were calculated twice by the same investigator at an interval of about 3 months (table 1). The degree of intra-observer variability was affected by the stage (end diastolic or end systolic) at which the volume was measured. Nevertheless, the first and second determination at both end systole and diastole were not statistically different (Student's paired $t$ test) $(\mathrm{p}<0.05)$.

\section{Results}

Table 2 shows the calculated ventricular volumes and derived functional indices in the three groups of patients. There was no statistically significant difference between the left ventricular end diastolic volume of the postextrasystolic $(\mathrm{P})$ beat and the control $(\mathrm{C})$ beat in all three groups. The only pre-extrasystolic values that were significantly different from those in the controls were the increased end diastolic and end systolic volumes and decreased ejection fraction found in group 3.

Although the end diastolic volume preceding the post-extrasystolic beat was unchanged and the contribution of a compensatory pause was excluded, several indices of left ventricular function indicated enhanced performance of

Table 2 Mean (1SD) calculated ventricular volumes and derived functional indices

\begin{tabular}{lllllll}
\hline & & & & \multicolumn{2}{l}{ Coefficients of: } \\
\cline { 5 - 7 } Volume & $n$ & Mean & Difference & $S D$ & Correlation $(r)$ & Variation $(\%) \dagger$ \\
\hline End diastolic & 53 & 77 & 0.53 & 5.4 & 0.98 & 7 \\
End systolic & 36 & 42 & 0.94 & 7.1 & 0.96 & 17 \\
\hline
\end{tabular}

^ Mean difference between the first and the second determinations.

$+\frac{\mathrm{SD}}{\text { mean }} \times 100^{\prime \prime}$

The first and second determinations of end diastolic and end systolic volumes were not significantly different.

\begin{tabular}{|c|c|c|c|c|c|c|c|}
\hline & \multicolumn{2}{|c|}{ Group $1(n=11)$} & \multicolumn{2}{|c|}{ Group $2(n=19)$} & \multicolumn{2}{|c|}{ Group $3(n=30)$} & \multirow[b]{2}{*}{$p$} \\
\hline & Pre & Post & Pre & Post & Pre & Post & \\
\hline $\begin{array}{l}\text { Ejection fraction } \\
\text { MNSER } \\
\text { End systolic volume } \\
\text { Stroke volume } \\
\text { End diastolic volume }\end{array}$ & $\begin{array}{l}0.64(0 \cdot 10) \\
147(35) \\
24(8) \\
40(6) \\
64(8)\end{array}$ & $\begin{array}{l}0.66(0 \cdot 10) \mathrm{p}<0.05 \\
150(38) \\
23(9) \\
42(6) \mathrm{p}<0.05 \\
66(9)\end{array}$ & $\begin{array}{l}0.64(0 \cdot 10) \\
155(39) \\
25(12) \\
43(9) \\
68(17)\end{array}$ & $\begin{array}{l}0.66(0.13) \\
164(44) \mathrm{p}<0.05 \\
24(14) \\
45(10) \\
69(17)\end{array}$ & $\begin{array}{l}0 \cdot 46(0 \cdot 12) \\
142(44) \\
51(23) \\
40(11) \\
91(24)\end{array}$ & $\begin{array}{l}0.52(0 \cdot 13) \\
166(65) \\
45(22) \\
46(14) \\
91(26)\end{array}$ & $\begin{array}{l}<0.05 \\
<0.05 \\
<0.05 \\
<0.05\end{array}$ \\
\hline $\begin{array}{l}\text { Basic interval (ms) } \\
\text { Extrasystolic } \\
\text { interval (ms) }\end{array}$ & 754 & & \multicolumn{2}{|c|}{$533(71)$} & \multicolumn{2}{|c|}{$559(76)$} & \\
\hline$\frac{\text { Extrasystolic }}{\text { basic }} \times 100$ & \multicolumn{2}{|c|}{$72(5)$} & \multicolumn{2}{|c|}{$72(6)$} & 75 & & \\
\hline Age (yr) & \multicolumn{2}{|c|}{$50 \cdot 0 \quad(8)$} & \multicolumn{2}{|c|}{$52 \cdot 4$} & \multicolumn{2}{|c|}{$50.9(10)$} & \\
\hline
\end{tabular}

MNSER, mean normalised systolic ejection rate.

There was strong evidence of potentiation in all four systolic variables in the group of patients with documented transmural myocardial infarction (group 3 ). 
that beat (table 2). In the post-extrasystolic beat the end systolic volume was decreased; ejection fraction, mean normalised ejection rate, and stroke volume were increased significantly in group 3. Thus the degree of potentiation was greatest in the group with preexisting left ventricular dysfunction. In contrast, the potentiation effects in groups 1 and 2 were slight or absent (table 2).

\section{Discussion}

We found that volume derived variables indicated little or no post-extrasystolic potentiation of left ventricular performance in controls and in patients with coronary heart disease without myocardial infarction. In these two groups only one of the three indices of left ventricular performance indicated the presence of post-extrasystolic potentiation. In patients with previous myocardial infarction, however, potentiation was evident in all the measured indices and reference values for left ventricular function were abnormal (table 2). This potentiation was independent of preload - as it was in healthy individuals in an earlier study ${ }^{12}$ and in animals. ${ }^{17}$ Potentiation may be attributed to an increased contractile reserve in non-infarcted areas, which compensates for the loss of contractile power in the infarcted myocardium. Van der Werf et al reported post-extrasystolic potentiation of the left ventricular performance when they used the same stimulation protocol in healthy individuals and in patients with coronary heart disease without infarction. ${ }^{14}$ They were studying pressure derived variables, and they too found no significant difference between the preloads of the post-extrasystolic beats and the pre-extrasystolic beats.

In most studies ${ }^{18-20}$ the contribution of the prolonged pause after an extrasystole to the enhanced myocardial contractile performance was attributed to an increase in end diastolic volume and an increase in the active state mediated by calcium. ${ }^{21}$ These two effects were avoided in the present study by preventing augmented diastolic filling and by keeping the post-extrasystolic interval equal to the basic interval. So the Frank-Starling mechanism ${ }^{22}$ and/or "rest contraction" "8-10 do not necessarily participate in or contribute to the increase of the post-extrasystolic contraction.

The role of the compensatory pause and its contribution as part of the interval-force relation to the increased performance of the post-extrasystolic beat has been overlooked by many investigators of post-extrasystolic potentiation. ${ }^{4121318-202324}$ In these studies the so-called post-extrasystolic beat is a beat potentiated by the prepreceding shorter interval and at the same time augmented by the longer interval that immediately preceded it. ${ }^{8}$ Sung et al showed post-extrasystolic potentiation with a compensatory pause in healthy individuals-as in our study without an increased left ventricular volume. ${ }^{12}$ Compar- ison of the results of this study with ours emphasises the contribution of the prolonged pause after the extrasystole to the increase in the force of the post-extrasystolic beat.

Our study of volume derived indices showed that post-extrasystolic potentiation of the left ventricle did not depend on the preload. Our results suggest that the Frank-Starling mechanism is not involved in post-extrasystolic potentiation in normal and diseased human hearts.

1 Langendorff $\mathrm{O}$. Über die elektrische Reizung des Herzens. Arch Anat Physiol 1885;8:284-7.

2 Hoffman BF, Bindler E, Suckling EE. Postextrasystolic potentiation of contraction Physiol 1956;185:95-102.

3 Cohn PF. Evaluation of inotropic contractile reserve in ischemic heart disease using postextrasystolic potentia tion. Circulation 1980;61:1071-5.

4 Hamby RI, Aintablian A, Wisoff BG, Hartstein ML. Response of the left ventricle in coronary artery disease to postextrasystolic potentiation. Circulation 1976;51: 428-35.

5 Braunwald E, Ross J Jr, Sonnenblick EH. Mechanisms of contraction of the normal and failing heart. 2nd ed. Boston: Little, Brown, 1976

6 Banka VS, Bodenheimer MM, Shah R, Helfant RH Intervention ventriculography. Comparative value of nitroglycerin, postextrasystolic potentiation and nitroglycerin plus postextrasystolic potentiation. Circulation 1976;53:632-7.

7 Beck W, Chesler E, Schrire V. Postextrasystolic ventricular pressure responses. Circulation 1971;44:523-33.

8 Meijler FL. Staircase, rest contractions, and potentiation in the isolated rat heart. Am J Physiol 1962;202:636-40.

9 Seed WA, Walker JM. Relation between beat interval and force of the heartbeat and its clinical implications. Cardiovasc Res 1988;22:303-14.

10 Meijler FL, Kuijer PJP, Van Dam-Koopman I, Heethaa RM, Van der Werf T. Clinical relevance of postextrasystolic potentiation. Mayo Clin Proc 1982;57(suppl) 34-9.

11 Kruta V, Braveny P. Restitution de la contractilité du myocarde entre les contractions et les phénomènes de potentiation. Arch Int Physiol 1961;69:645-67.

12 Sung CS, Mathur VS, Garcia E, De Castro CM, Hall RJ. Is postextrasystolic potentiation dependent on Starling's postextrasystolic potentiation depe
law? Circulation 1980;62:1032-5.

13 Wisenbaugh Th, Nissen S, DeMaria A. Mechanics of postextrasystolic potentiation in normal subjects and postextrasystolic potentiation in normal subjects and 1986;74:10-20.

14 Van der Werf T, Van Poelgeest R, Herbschleb JN, Meijle FL. Postextrasystolic potentiation in man. Eur $J$ Cardio 1976;4 (suppl):131-41.

15 Marier DL, Gibson DG. Limitations of two frame method for displaying regional left ventricular wall motion in man Br Heart J 1980;44:555-9.

16 Dodge HT, Sandler H, Ballew DW, Lord JD Jr. The use of biplane angiography for the measurement of left ventricular volume in man. Am Heart $J 1960 ; 60: 762-76$.

17 Kuijer PJP, Heethaar RM. Herbschleb JN, extrasystolic relaxation in the dog heart. Eur $J$ Cardiol 1978;7 (suppl):133-45.

18 Klausner SC, Ratshin RA, Tyberg JV, Lappin HA Chatteriee K, Parmley WW. The similarity of changes in segmental contraction patterns induced by post-extrasegmental contraction patterns induced by post-extrasystolic potentiatic

19 White CW, Zimmerman TJ. Prolonged left ventricular ejection time in the post-premature beat: a sensitive sign of idiopathic hypertrophic subaortic stenosis. Circulation 1975;52:306-12.

20 Cohn PF, Angoff GH, Sloss LJ, et al. Noninvasively induced postextrasystolic potentiation of ischemic and infarcted myocardium in patients with coronary artery disease. Am Heart J 1979;97:187-94.

21 Edman KAP, Johansson $M$. The contractile state of rabbit papillary muscle in relation to stimulation frequency. J Physiol (Lond) 1976;254:565-81.

22 Starling EH. The Linacre lecture on the law of the heart. London: Longmans, Green, 1918.

23 Diamond GA, Forrester JS, De Luz PL, Wyatt HL, Swan HJC. Postextrasystolic potentiation of ischemic myocarHJC. Postextrasystolic potentiation of ischemic myoca
dium by atrial stimulation. Am Heart J 1978;95:204-9.

24 Popio KA, Gorlin R, Bechtel D, Levine JA. Postextrasystolic potentiation as a predictor of potential myocardial viability: preoperative analysis compared with studie after coronary bypass surgery. Am J Cardiol 1977;39: 944-53. 